\title{
Magnetic domain structure of nanohole arrays in Ni films
}

\author{
M. Jaafar, D. Navas, A. Asenjo, and M. Vázquez \\ Instituto de Ciencia de Materiales de Madrid, ICMM-CSIC, Cantoblanco, 28049 Madrid, Spain \\ M. Hernández-Vélez \\ Departamento de Física Aplicada, Universidad Autónoma de Madrid, Cantoblanco, 28049 Madrid, Spain \\ J. M. García-Martín ${ }^{a)}$ \\ Instituto de Microelectrónica de Madrid, IMM-CSIC, Isaac Newton 8, Tres Cantos, 28760 Madrid, Spain
}

(Presented on 9 January 2007; received 31 October 2006; accepted 13 December 2006; published online 2 May 2007)

\begin{abstract}
Nanohole arrays in Ni films have been prepared by a replica/antireplica method based on anodic alumina membranes. The nanohole arrays exhibited long range ordering with hexagonal symmetry, the hole distance was kept constant $(105 \mathrm{~nm})$, and the hole diameter and the film thickness were varied between 50 and $70 \mathrm{~nm}$ and 55 and $600 \mathrm{~nm}$, respectively. The magnetic domain structures of such samples have been studied by analyzing magnetic force microscopy images at remanent state. Different domain structures have been observed depending on the geometrical characteristics of the films. The experimental results have been interpreted with the help of micromagnetic simulations. (c) 2007 American Institute of Physics. [DOI: 10.1063/1.2711613]
\end{abstract}

\section{INTRODUCTION}

The development of nanostructured materials opens the opportunity to study new physical properties which are not present in bulk. For the past years several groups have done great effort in the fabrication of new systems such as nanowires, ${ }^{1,2}$ nanotubes, ${ }^{3}$ nanorings,${ }^{4}$ nanoparticles, ${ }^{5}$ dots, ${ }^{6}$ antidots, ${ }^{7}$ etc., in most cases by using lithographic techniques. However, the preparation methods based on the use of templates are efficient and inexpensive methods. Nanoporous anodic alumina membranes (AAMs) are commonly used as templates for the fabrication of ordered nanomaterials and particularly for the preparation of nanowires and nanotube arrays $^{8}$ with hexagonal symmetry. In the recent years, our work has been focused in the growth of magnetic nanowire arrays by electrodeposition using AAMs as templates. ${ }^{9,10}$

The preparation of metallic membranes by replicaantireplica methods by using AAMs as templates was first reported by Masuda and Fukuda. ${ }^{11}$ In that early work, Pt and $\mathrm{Au}$ nanohole arrays were prepared with a pore diameter of $70 \mathrm{~nm}$. Recently, the same group has fabricated arrays of $\mathrm{Ni}$ nanoholes with $300 \mathrm{~nm}$ diameter and $500 \mathrm{~nm}$ interhole distance. ${ }^{12}$ In previous works, ${ }^{13,14}$ we have reported the fabrication method (based on a modified Masuda's technique) of Ni nanohole arrays with $105 \mathrm{~nm}$ separation between adjacent nanoholes, pore diameters between 35 and $70 \mathrm{~nm}$, and different thicknesses. Our samples are almost perfect replicas of the AAMs used as templates.

It is worth mentioning that nanohole arrays in magnetic thin films are interesting both from a fundamental point of view (in order to understand the micromagnetic behavior of these exceptional systems) and also for developing advanced sensing devices and a new generation of data storage media based on domain wall structures. ${ }^{7}$

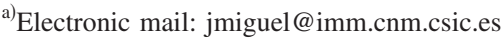

The objective of the present work is to study the domain structure of these magnetic membranes. Due to the low dimension of the system, it is necessary to use a high resolution technique such as the magnetic force microscope (MFM). The experimental results are interpreted using micromagnetic simulations.

\section{EXPERIMENTAL DETAILS}

Nanoporous alumina films with long range hexagonal ordering have been first prepared by a two-step anodization process of high purity (99.999\%) aluminum foils to be used as template for subsequent processing (details can be found in Refs. 9 and 15). The original alumina membrane has thickness of about $45 \mu \mathrm{m}$, pore diameter around $35 \mathrm{~nm}$, and lattice parameter of long range hexagonal symmetry of about $105 \mathrm{~nm}$. In order to prepare AAM templates with larger pore diameter, the alumina membrane was immersed in a $5 \mathrm{wt} \%$ phosphoric acid aqueous solution: depending on the duration of the acid treatment, AAMs with 50,60, and $70 \mathrm{~nm}$ pore diameters were obtained.

The replica/antireplica processes consisted of various steps that can be summarized in two: (1) obtaining a polymethyl methacrylate (PMMA) antireplica by pressure and (2) growing a metal $(\mathrm{Ni})$ replica by electrodeposition. Following the detailed method shown in Refs. 13 and 14, the ordering of the AAM precursor is transferred into the final metallic membrane. Thus, metallic membranes with pore diameters of 50,60 , and $70 \mathrm{~nm}$ have been obtained. It is worth noting that the samples are submitted to a small magnetic field during step 2 since the electrolytical cell has a rotating magnetic agitator.

A microscope from Nanotec Electrónica S.L. working at ambient conditions has been used for the MFM imaging. The topography of the surface and the magnetic force gradient map, which is proportional to the magnetic pole density, are 

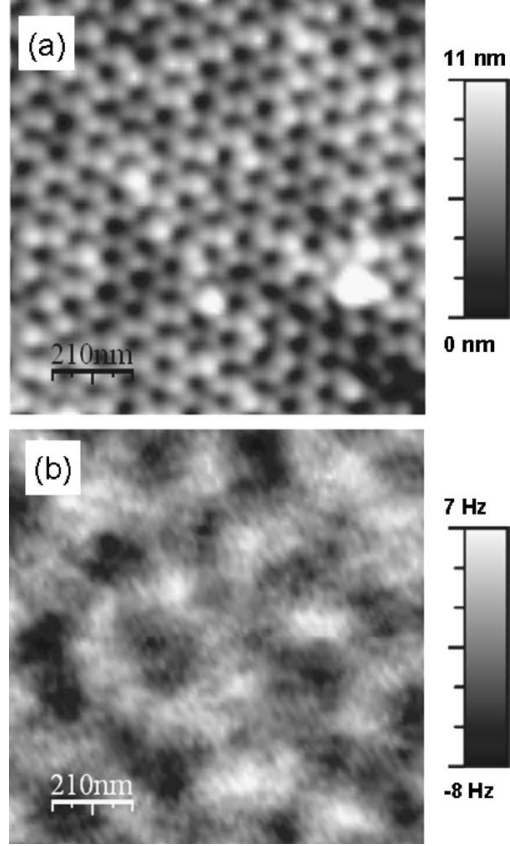

FIG. 1. Topography (a) and magnetic (b) images obtained by MFM of a nanohole array (hole diameter: $50 \mathrm{~nm}$ ) in a $55 \mathrm{~nm}$ thick Ni film.

acquired simultaneously in noncontact mode. Low moment cantilevers from Veeco with a force constant of $K_{c}$ $\approx 5 \mathrm{~N} / \mathrm{m}$ and $\mathrm{CoCr}$ tip radius of $\mathrm{R} \approx 20 \mathrm{~nm}$ have been used to prevent a strong influence of the tip stray field on the sample.

Micromagnetic simulations using the OOMMF $\operatorname{code}^{16}$ have been made with the typical parameters for $\mathrm{Ni}$ : exchange constant $A=9 \times 10^{-12} \mathrm{~J} / \mathrm{m}$, saturation magnetization $M_{S}$ $=4.9 \times 10^{5} \mathrm{~A} / \mathrm{m}$, zero magnetocrystalline anisotropy (polycrystalline sample), and cell size of $4 \mathrm{~nm}$ (i.e., below the exchange length for $\mathrm{Ni}$ ). Suitable bitmap files were prepared and used as masks to define similar structures with those experimentally observed. In order to remove as much as possible the influence of the finite size of the simulated samples, arrays with about 600 nanoholes have been used, and the results shown below correspond to the central region of the array. In order to compare with the experimental MFM images that give a map of the magnetic poles at the sample surface, the magnetic poles of the simulated magnetization distributions have been properly coded in a gray scale.

\section{RESULTS AND DISCUSSION}

In order to obtain information on the magnetic behavior and, particularly, on the magnetic domain structure, MFM imaging of the sample surface has been performed in the as-prepared state and in the remanent state after an in-plane hysteresis loop, giving rise to similar results except where is mentioned later.

In Fig. 1, the topography [Fig. 1(a)] and magnetic contrast [Fig. 1(b)] images of the Ni membrane with $50 \mathrm{~nm}$ pore diameter and $55 \mathrm{~nm}$ thick are shown. In this case, according to the hysteresis loops (not shown, see Refs. 13 and 14; the in-plane and perpendicular remanent magnetizations are 0.35 and 0.06 , respectively), the magnetization is mainly in plane. However, by MFM, dark and bright regions can be distin-
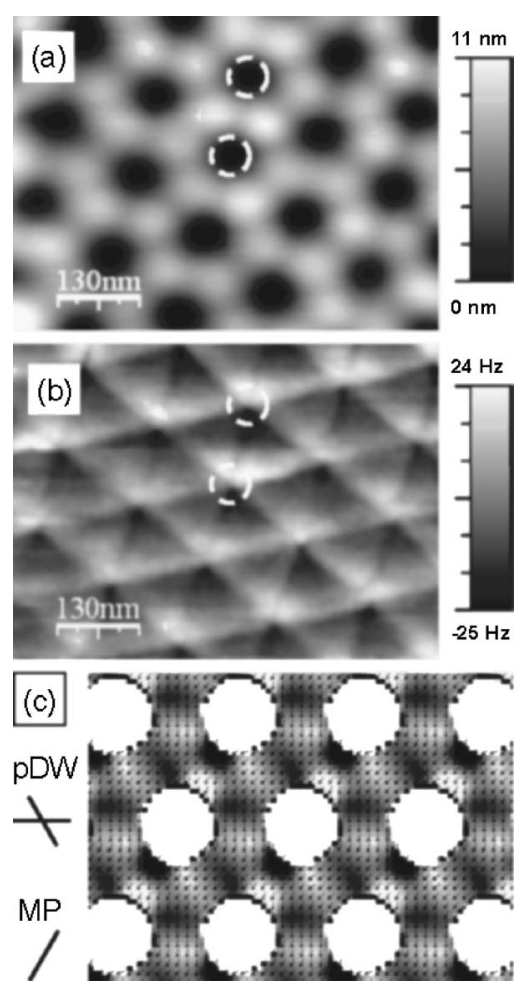

FIG. 2. Topography (a) and magnetic (b) images obtained by MFM of a nanohole array (hole diameter: $60 \mathrm{~nm}$ ) in a $200 \mathrm{~nm}$ thick Ni film. The dotted circles indicate the position of two holes for comparison. Micromagnetic simulation (c) of such array exhibiting magnetic poles accumulated at the holes along the MP direction and pseudodomain walls along the pDW directions; the gray scale represents the magnetization divergence, i.e., the magnetic poles.

guished in the magnetic image without correlation to the topography. As the lateral size of these regions is of the order of magnitude of the sample thickness and their orientation is not related to the field direction during the loop, such contrast can be ascribed to a small out of plane component which oscillates in up and down directions to reduce the magnetostatic energy (similar to the dense stripe domains ${ }^{17}$ ), creating magnetic poles of alternating signs that give rise to the dark and bright regions in the magnetic image.

When the hole diameter and the thickness of the film increase to 60 and $200 \mathrm{~nm}$ respectively, the magnetic distribution observed by MFM drastically changes [Figs. 2(a) and 2(b)]. In this case, the topography and the magnetic images are correlated due the pinning effect that is more important when the diameter of the hole increases, as deduced from the hysteresis loops. ${ }^{13,14}$ By analyzing the MFM images, it can be found that of the three directions that define the hexagonal symmetry, an accumulation of magnetic charges around the nanoholes can be observed along one of them, while straight lines connecting adjacent nanoholes can be distinguished along the other two directions (the nanoholes themselves are not distinguished in the magnetic image due to the convolution with the effective magnetic volume of the tip of the microscope). With the help of micromagnetics simulations, one can understand such contrast. If an initial in-plane field close to a symmetry axis is assumed in the simulations, the remanent state is that depicted in Fig. 2(c): the in-plane magnetization is along the magnetic field direction (labeled MP) 


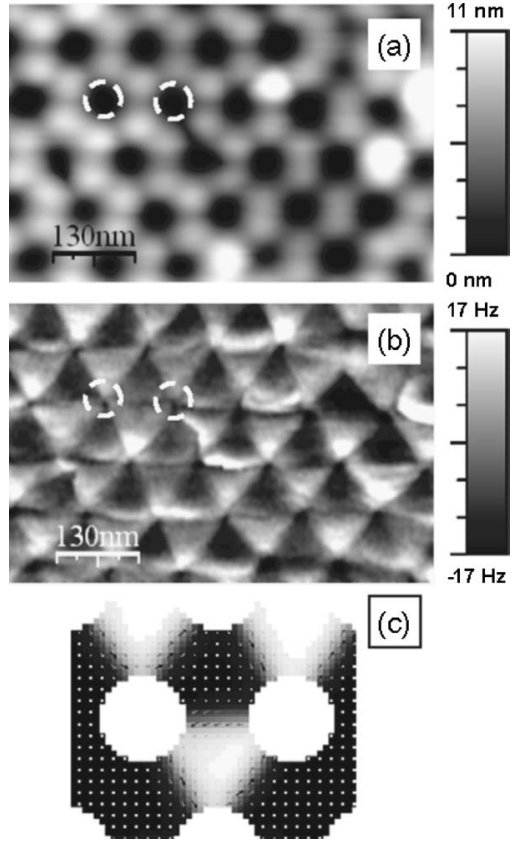

FIG. 3. Topography (a) and magnetic (b) images obtained by MFM of a nanohole array (hole diameter: $60 \mathrm{~nm}$ ) in a $300 \mathrm{~nm}$ thick Ni film. The dotted circles indicate the position of two holes for comparison. Micromagnetic simulation (c) of such array exhibiting perpendicular magnetization; the gray scale represents the out of plane component of the magnetization, responsible for the magnetic poles at the surface.

accumulating magnetic poles of opposite sign at each side of the nanohole. Moreover, there are pseudodomain walls pinned at the nanoholes along the other two directions connecting neighboring holes (labeled pDW).

Finally, a different magnetic structure [Figs. 3(a) and $3(b)]$ appears in the sample with a larger thickness $(300 \mathrm{~nm})$ and similar hole diameter $(60 \mathrm{~nm})$ in the as-prepared state. In this case, a magnetic board with triangular-shaped structures can be distinguished, with the triangle sides located along the three privileged directions connecting neighboring holes. The perpendicular hysteresis loops (not shown, see Ref. 14) exhibited a saturation field of about $2 \mathrm{kOe}$, smaller than that obtained for an electrodeposited continuous $\mathrm{Ni}$ film with similar thickness $(4.6 \mathrm{kOe})$, pointing to some perpendicular anisotropy for the array. In fact, triangular-shaped domains can be obtained in the simulations if a perpendicular anisotropy of $10^{5} \mathrm{~J} / \mathrm{m}^{3}$ is assumed [Fig. 3(c)]. In this situation, each triangle corresponds to a magnetic domain with a strong perpendicular magnetization component. Such perpendicular anisotropy cannot be ascribed to magnetocrystalline anisotropy: the samples are polycrystalline, and even a strong (111) texture would produce an anisotropy of around 6 $\times 10^{3} \mathrm{~J} / \mathrm{m}^{3}$ only. Our guess is that such anisotropy is due to the sample geometry, in particular, to the corrugation of several nanometers [i.e., the valley lines connecting neighboring holes that can be distinguished in Fig. 3(a)] that can act as pinning lines for domain walls. Furthermore, the fact that the thickness $(300 \mathrm{~nm})$ is three times larger than the distance between adjacent hole centers $(105 \mathrm{~nm})$ can also favor per- pendicular magnetization. This shape anisotropy can be of the order of $0.5 \mu_{0} \times M_{s}{ }^{2} \sim 1.6 \times 10^{5} \mathrm{~J} / \mathrm{m}^{3}$ for Ni. Moreover, it is worth noting that similar triangular-shaped domains were observed for a sample with $600 \mathrm{~nm}$ thickness and $70 \mathrm{~nm}$ hole diameter. The only difference is that the magnetic contrast, which is $\pm 17 \mathrm{~Hz}$ for the $300 \mathrm{~nm}$ thick sample shown in Fig. 3, became $\pm 38 \mathrm{~Hz}$ for the $600 \mathrm{~nm}$ thick one, in agreement with the increase of magnetic volume in each domain. Finally, it has to be mentioned that a domain structure similar to that shown in Fig. 2 can also be obtained in these samples after an in-plane hysteresis loop. Consequently, the magnetic state of the thickest samples depends on their magnetic history also.

\section{CONCLUSSIONS}

Nanohole arrays in Ni films exhibiting long range ordering of hexagonal symmetry with $105 \mathrm{~nm}$ periodicity have been studied by combining MFM imaging and micromagnetic simulations. Two key parameters govern the domain structure of the arrays: the hole diameter and the film thickness. Pore diameters of $60 \mathrm{~nm}$ and above pin the domain walls. Film thickness above $250 \mathrm{~nm}$ can induce perpendicular anisotropy.

\section{ACKNOWLEDGMENTS}

This work has been supported by the following institutions: CSIC under the project BIOPTOMAG, CAM under the program NANOMAGNET (S-0505/MAT/0194), and MEC under Project No. NAN2004-09183-C10-04. One of the authors (M.J.) thanks CAM for her postgraduate fellowship.

${ }^{1}$ K. Nielsch, F. Müller, A.-P. Li, and U. Gösele, Adv. Mater. (Weinheim, Ger.) 12, 582 (2000)

${ }^{2}$ L. Piraux, A. Encinas, L. Vila, S. Mátéfi-Tempfli, M. Mátéfi-Tempfli, M. Darques, F. Elhoussine, and S. Michotte, J. Nanosci. Nanotechnol. 5, 372 (2005).

${ }^{3}$ D. Mendoza, Opt. Mater. (Amsterdam, Neth.) 29, 122 (2006).

${ }^{4}$ F. J. Castaño, D. Morecroft, W. Jung, and C. A. Ross, Phys. Rev. Lett. 95, 137201 (2005).

${ }^{5}$ J. Aumentado and V. Chandrasekhar, Physica B 284-288, 1742 (2000).

${ }^{6}$ T. J. Bromwich, A. Kohn, A. K. Petford-Long, T. Kasama, R. E. DuninBorkowski, S. B. Newcomb, and C. A. Ross, J. Appl. Phys. 98, 053909 (2005).

${ }^{7}$ R. P. Cowburn, A. O. Adeyeye, and J. A. C. Bland, Appl. Phys. Lett. 70, 2309 (1997).

${ }^{8}$ S. Shingubara, J. Nanopart. Res. 5, 17 (2003).

${ }^{9}$ M. Vázquez, K. R. Pirota, M. Hernández-Vélez, V. M. Prida, D. Navas, R. Sanz, F. Batallán, and J. Velázquez, J. Appl. Phys. 95, 6642 (2004).

${ }^{10}$ D. Navas et al., J. Magn. Magn. Mater. 290-291, 191 (2005).

${ }^{11}$ H. Masuda and K. Fukuda, Science 268, 1466 (1995).

${ }^{12}$ T. Yanagishia, K. Nishio, and H. Masuda, Adv. Mater. (Weinheim, Ger.) 17, 2241 (2005).

${ }^{13}$ D. Navas, M. Hernández-Vélez, A. Asenjo, M. Jaafar, J. L. Baldonedo, and M. Vazquez, IEEE Trans. Magn. 42, 3057 (2006).

${ }^{14}$ D. Navas, M. Hernández-Vélez, W. Lee, and M. Vazquez (unpublished).

${ }^{15}$ A.-P. Li, F. Müller, A. Birner, K. Nielsch, and U. Gösele, Adv. Mater. (Weinheim, Ger.) 11, 483 (1999).

${ }^{16}$ M. J. Donahue and D. J. Porter, http://math.nist.gov/oommf/

${ }^{17}$ A. Asenjo, D. García, J. M. García, C. Prados, and M. Vázquez, Phys. Rev. B 62, 6538 (2000). 